\title{
Do we really need to invoke heroic measures for early SARS-CoV-2 outbreak detection?
}

\author{
Gorka Orive $e^{1,2,3,4}$ (1) $\cdot$ Unax Lertxundi ${ }^{5} \cdot$ Damiá Barceló $^{6,7}$
}

Received: 20 May 2020 / Accepted: 5 June 2020 / Published online: 11 June 2020

C) Springer Nature B.V. 2020

Over half of the world's population was locked down due to the SARS-CoV-2 pandemic [1]. Countries are gradually easing lock-down restrictions, allowing citizens to recover their new "normal life", while authorities prepare for a possible second wave.

In these times of uncertainty, government policies around the world are based on model assumptions rather on realtime evidence, as traditional epidemiological tools face important logistic and feasibility challenges. The importance of asymptomatic carriers in the epidemiology of COVID-19 is driving scientists to consider massive testing in order to promptly detect re-emergence of SARS-CoV-2 outbreaks in communities. For example, a "Dunkirk spirit" is called as the only way to perform 10 million PCR tests per day in the United Kingdom, so that every citizen is voluntarily weekly tested [2]. But if people who test positive know they will be

Gorka Orive

gorka.orive@ehu.eus; gorka.orive@ehu.es

1 Laboratory of Pharmaceutics, NanoBioCel Group, School of Pharmacy, University of the Basque Country UPV/EHU, Paseo de la Universidad 7, 01006 Vitoria-Gasteiz, Spain

2 Biomedical Research Networking Centre in Bioengineering, Biomaterials and Nanomedicine (CIBER-BBN), Vitoria-Gasteiz, Spain

3 University Institute for Regenerative Medicine and Oral Implantology - UIRMI (UPV/EHU-Fundación Eduardo Anitua), Vitoria-Gasteiz, Spain

4 Singapore Eye Research Institute, The Academia, 20 College Road, Discovery Tower, Singapore, Singapore

5 Bioaraba Health Research Institute, Osakidetza Basque Health Service, Araba Mental Health Network, Araba Psychiatric Hospital, Pharmacy Service, Vitoria-Gasteiz, Spain

6 Water and Soil Quality Research Group, Department of Environmental Chemistry, IDAEA-CSIC, C/Jordi Girona 18-26, 08034 Barcelona, Spain

7 Catalan Institute for Water Research (ICRA), C/Emili Grahit 101, 17003 Girona, Spain put back into quarantine, the voluntariness may be a pitfall of this ambitious plan.

We believe the key for a complementary valid strategy could be found in a recently study published by Zheng et al. where a median duration of SARS CoV-2 virus in stool of approximately three weeks was observed [3]. Measuring virus presence in sewage has been sensitive enough to detect virus RNA presence before the first cases were documented through the Dutch health epidemiological surveillance system [4] and to reveal that the increase in SARS-CoV-2 genome units was followed by the increase in the number of deaths registered at both the regional and national levels in France [5]. So, a strategy based on continuous monitoring of wastewater may early alert public health officials of future coronavirus outbreaks. Considering that prolonged fecal shedding from past cases could 'mask' the signal from new cases, a thorough approach will remain necessary to avoid false alarms.

Sewage-based epidemiology offers one obvious advantage. Each person sample's no longer need to be collected, transported and individually analyzed. Instead, all wastewater treatment plants catchment population's sample will come to you via sewage collector pipes. Effort on human clinical testing could be then strengthened in identified hot spots. In addition, this method could be useful in developing countries where resources for clinical diagnosis are limited. However, as many cities from the Global South lack proper wastewater treatment, collecting representative population samples may prove difficult. Besides, pressure to use the scarce available resources for clinical testing may be high.

We hypothesize whether this approach might also be explored at a more localized context, especially on nursing homes, which have been proven a blind spot in many countries [6]. In addition, novel technological platforms such as paper analytical devices could shed light on the cost-efficient detection of the coronavirus nucleic acid sequences [7].

On the other hand, a recent case report from China has shown infectious SARS-CoV-2 virus in the feces of a 
severely ill patient, affirming the potential for fecal-oral or fecal-respiratory transmission through aerosolized feces [8]. If this hypothesis is confirmed, additional measures may be necessary to help mitigate the dissemination of the disease. Besides, a careful risk-management assessment for both the water and health workers would be necessary. However, some authors believe that, still, a more comprehensive and nuanced analysis is needed to test this hypothesis, taking into consideration both environmental dynamics and the persistence of viral infectivity [9]. In addition, a cautious approach will be required when informing public opinion on this issue, since suggested COVID-19 risks could drive people away from basic hygienic measures (hand-washing) that are critical in limiting the transmission of this coronavirus.

In short, sewage epidemiology of the SARS-CoV-2 virus will reveal trends of the pandemic outbreak providing health authorities with complementary, cheap and fast risk-management solutions.

\section{References}

1. Half of the world in lock down. Available at: https://www.daily mail.co.uk/news/article-8181001/3-9-billion-people-currentlycalled-stay-homes-coronavirus.html. Accessed 4 June 2020.

2. Peto J, Alwan NA, Godfrey KM, Burgess RA, Hunter DJ, Riboli E, et al. Universal weekly testing as the UK COVID-19 lockdown exit strategy. Lancet. 2020. https://doi.org/10.1016/S0140 -6736(20)30936-3.

3. Zheng S, Fan J, Feng B, Lou B, Zou Q, Xie G, Lin S, Wang R, Yang X, Chen W, Wang Q, Zhang D, Liu Y, Gong R, Ma Z, Lu S, Xiao Y, Gu Y, Zhang J, Yao H, Xu K, Lu X, Wei G, Zhou J, Fang Q, Cai H, Qiu Y, Sheng J, Chen Y, Liang T. Viral load dynamics and disease severity in patients infected with SARS-CoV-2 in Zhejiang province, China, January-March 2020: retrospective cohort study. BMJ. 2020;369:m1443. https://doi.org/10.1136/bmj. m1443.

4. Medema G, Heijnen L, Elsinga G, Italiaander R, Brouwer A. Presence of SARS-Coronavirus-2 in sewage. MedRxiv. 2020. https:// doi.org/10.1101/2020.03.29.20045880.

5. Wurtzer S, Marechal V, Mouchel JM, Moulin L. Time course quantitative detection of SARS-CoV-2 in Parisian wastewaters correlates with COVID-19 confirmed cases. MedRxiv. 2020. https ://doi.org/10.1101/2020.04.12.20062679.

6. Rada AG. Covid-19: the precarious position of Spain's nursing homes. BMJ. 2020:369:m1554. https://doi.org/10.1136/bmj. $\mathrm{m} 1554$.

7. Reboud J, Xu G, Garrett A, Adriko M, Yang Z, Tukahebwa EM, Rowell C, Cooper JM. Paper-based microfluidics for DNA diagnostics of malaria in low resource underserved rural communities. Proc Natl Acad Sci USA. 2019;116(11):4834-42. https://doi. org/10.1073/pnas.1812296116.

8. Xiao F, Sun J, Xu Y, Li F, Huang X, Li H, Zhao J, Huang J, Zhao J. Infectious SARS-CoV-2 in Feces of Patient with Severe COVID-19. Emerg Infect Dis. 2020. https://doi.org/10.3201/eid26 08.200681.

9. Heller L, Mota CR, Greco DB. COVID-19 faecal-oral transmission: Are we asking the right questions? Sci Total Environ. 2020;729:138919. https://doi.org/10.1016/j.scitotenv.2020.13891 9. 